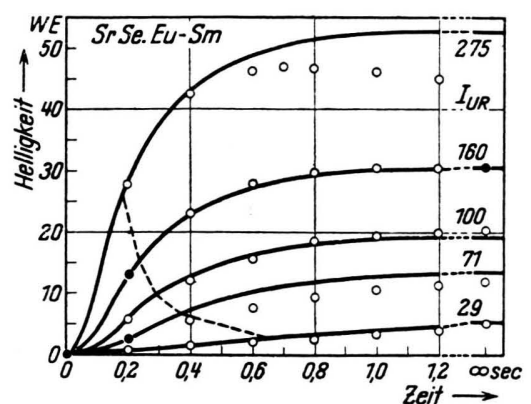

Abb. 2. Anfang des zeitlichen Verlaufs der Helligkeit der Lichtemission von erregtem SrSe-Eu-Sm unter bei $t=0$ einsetzender konstanter Ultrarotbestrahlung der Intensität $I_{\mathrm{TR} \text {. }}$. Theoretische Kurven. Eingetragene Meßpunkte nach Scott, Thompson und Ellickson. Die Konstanten in den theoretischen Formeln wurden aus den vier ausgefüllten Punkten bestimmt. Die starke Abweichung der Kurve $I_{\mathrm{IR}}=275$ rührt von der in der Theorie nicht berücksichtigten merklichen Entleerung der Sm-Haftstellen her, die der Kurve $I_{\mathrm{TR}}=71$ wahrscheinlich von einem Meßfehler, wie man am Endwert erkennt.

abstände usw.) sind mit den für die betreffenden Kristalle zu erwartenden in Einklang, wenn auch ein genauerer Vergleich mit den spektroskopisch oder aus dem thermischen Verhalten bestimmten Werten sich noch kaum durchführen ließ, da es sich bei den von den genannten Autoren mitgeteilten Messungen im Grunde um Relativmessungen handelt.
Abb. 1 zeigt das zeitliche Anklingen der Helligkeit von erregtem SrS-Eu-Sm bei einsetzender Ultrarotbestrahlung. Hier sei besonders auf den plötzlichen Anstieg (Stufe), dessen Höhe der Ultrarotintensität im Gegensatz zum Endwert nicht proportional ist, hingewiesen. Abb. 2 zeigt Verhältnisse, wie sie nach Scott, Thompson und Ellickson beim SrSe-Eu-Sm verifiziert sind. Die Abwesenheit einer (größeren) Anfangsstufe deutet hier im Rahmen der Theorie darauf hin, daß das Ultrarot nur anregt und daß erst zusätzliche Wärmeenergie die Elektronen befreit. Ist dieser letztgenannte Prozeß von relativ sehr großer Wahrscheinlichkeit, dann entstehen Kurven vom Typus der Abb. 1. Darum könnte wahrscheinlich Temperaturerhöhung hier auch einen plötzlichen Anfangsanstieg wie in Abb. 1 hervorbringen. Das unterschiedliche Verhalten zwischen dem chemisch sehr ähnlichen SrS-Eu-Sm und SrSe-Eu-Sm wird nach unserer Theorie lediglich durch quantitative Unterschiede (Termabstände) hervorgerufen. Die nach Abschluß unserer Arbeit von R. C. H e r m a n u. Ch. F. M e y e ${ }^{3}$ mitgeteilte Theorie, die sich zudem mit einem qualitativen Vergleich begnügt, leistet dies, soweit der Verf. erkennen kann, in ihrer gegenwärtigen Form nicht.

Eine ausführliche Darstellung folgt demnächst.

3 R. C. H e rma n u. Ch. F. M e y e r, J. opt. Soc. America 39, 729 [1949].

\title{
Elektroakustische Messungen an berühmten Barockorgeln Oberschwabens II. Klanganalytische Untersuchungen
}

\author{
Von Werner LotTermoser \\ Aus dem Physikalischen Institut der Universität Tübingen \\ (Z. Naturforschg. 5 a, 159-168 [1950]; eingegangen am 21. November 1949)
}

\begin{abstract}
Es werden Klangspektren von Einzelpfeifen, besonders aber solche gebräuchlicher Pfeifenkombinationen, wie des Plenums und der Mixturen, wiedergegeben. Vergleichsmessungen an weniger wertvollen Klängen zeigen, daß die untersuchten Barockorgeln im Gesamtklang bestimmte, objektiv erfaßbare Gütemerkmale besitzen. Diese bestehen darin, daß gewisse Obertöne, die in einem breiten Frequenzbereich um $1000 \mathrm{~Hz}$ liegen, unabhängig von der Grundtonhöhe des Klanges stärker als die übrigen sind. Die günstige Intensitätsverteilung wird zum großen Teil bewirkt durch die gemischten Stimmen (Mixturen usw.), die wandernde Formanten erzeugen und dadurch den Orgelklang charakteristisch färben. Im vollen Klang aller Orgeln fällt die größere Intensität der Oktav- und Quintteiltöne auf.
\end{abstract}

$D^{-1}$ er 1 . Teil ${ }^{1}$ der Untersuchungen behandelte die Schalldrucke von Einzelregistern und ihrer Kombinationen und zeigte, daß zur Erzielung angenehmer Klangwirkungen die Einhaltung einer gewissen Schalld̊ruckgrenze und sorgfältig abgeglichene Schall-

1 W. Lottermoser, Z. Naturforschg. 3a, 298 [1949]. druckverhältnisse der Register untereinander notwendig sind. Der vorliegende 2. Teil soll Analysen von Einzel- und Gesamtklängen wiedergeben. Die hier wiedergegebenen Spektren der Einzelpfeifen sind als Ergänzung bereits vorliegender Ergebnisse?

$$
\text { 2W. Lottermoser, Akust. Z. 5, } 324 \text { [1940]. }
$$


hinsichtlich einer Erfassung der raumakustischen Einflüsse anzusehen, und die Spektren der Plenumklänge bringen neue Ergebnisse über die Intensitätsverteilung bei verschiedenen Frequenzen; es soll gezeigt werden, daß offenbar dem physiologisch angenehmen Klang eine bestimmte spektrale Verteilung zuzuordnen ist. Dies soll nicht im Sinne einer Normung verstanden werden, denn eine solche wäre bei dem vorwiegend künstlerischen Objekt fehl am Platze, sondern soll den zur Zeit oft recht undurchsichtigen Anschauungen über den Gegenstand klar durchschaubare, naturwissenschaftliche Beobachtungen gegenüberstellen. Damit wird der ganze Fragenkomplex zugänglicher und vornehmlich für den modernen Orgelbauer vereinfacht.

$\mathrm{Zu}$ den in Teil $1^{1}$ behandelten Barockorgeln ist die Gabler-Orgel in der Abteikirche Ochsenhausen (gebaut 1751 bis 1755) hinzugetreten. Diese Orgel gilt bei Kennern ${ }^{3}$ als die klanglich bessere von den beiden erhaltenen, großen Werken des Meisters, obwohl seine andere Orgel, im Kloster Weingarten, die bekanntere ist. Der klangliche Aufbau der GablerOrgel unterscheidet sich teilweise wesentlich von den bisher untersuchten Orgeln von $\mathrm{R}$ i e p p und $\mathrm{Holz}$ $\mathrm{h}$ ay. In der großen Zahl von Charakterstimmen drückt sich eine Abwendung von den Grundsätzen der klassischen, barocken Bauweise aus. So stellt dieses Instrument einen zur Abrundung vorliegender Ergebnisse bemerkenswerten Typ dar: Während Riepp bei seinen Instrumenten in Ottobeuren eine vollendete Gesamtwirkung und eine Fülle von Mischungsmöglichkeiten erzielte, Holzhay neben einer hervorragenden Synthese einzelne Charakterstimmen bot, legte Gabler den Hauptwert auf die Erzeugung einer Reihe von Einzelklangfarben, die ihm, das beweisen auch diese Untersuchungen, meisterhaft gelungen sind.

\section{M e $\beta$ methode}

Die Obertcnzusammensetzung der Klänge wurde teils durch Suchtonanalyse ${ }^{4}$, teils durch Oktavsiebanalyse 5 ermittelt. Beide Verfahren wurden in einigen Fällen direkt in der Kirche durchgeführt. Meistens wurden aber Magnetophonaufnahmen zur Analyse benutzt, da sich zeigte, da $\beta$ bei entsprechender Einrechnung der verschiedenen Übertragungsmaße die Ergebnisse praktisch die gleichen waren.

Für die Suchtonanalyse wurde das Hochtonprinzip mit

3 Der bekannte Orgelarchitekt Dr. W. Supper, Eßlingen, Sachbearbeiter des Landesamts für Denkmalpflege, hat diese Orgel zur Untersuchung vorgeschlagen; s. a. W. S u p per u. H. M e y e r, Barockorgeln in Oberschwaben, Bärenreiter-Verlag, Kassel.

\begin{tabular}{|c|rr|r|}
\hline & \multicolumn{2}{|c|}{ Stellung I } & \multicolumn{2}{|c|}{ Stellung II } \\
& $f_{\mathrm{u}}$ & $f_{\mathrm{u}}$ & $f_{\mathrm{u}}$ \\
\hline 1. & $50-100 \mathrm{~Hz}$ & $35-70 \mathrm{~Hz}$ \\
2. & $100-200 \mathrm{~Hz}$ & $70-141 \mathrm{~Hz}$ \\
3. & $200-400 \mathrm{~Hz}$ & $141-282 \mathrm{~Hz}$ \\
4. & $400-800 \mathrm{~Hz}$ & $282-564 \mathrm{~Hz}$ \\
5. & $800-1600 \mathrm{~Hz}$ & $564-1128 \mathrm{~Hz}$ \\
6. & $1600-3200 \mathrm{~Hz}$ & $1128-2256 \mathrm{~Hz}$ \\
7. & $3200-6400 \mathrm{~Hz}$ & $2256-4512 \mathrm{~Hz}$ \\
8. & $6400-12800 \mathrm{~Hz}$ & $4512-9024 \mathrm{~Hz}$ \\
\hline
\end{tabular}

Tab. 1. Filtergrenzen der benutzten Oktavsiebe.

Gegentaktmodulator und elektrisch entdämpftem Resonanzfilter angewandt. Die Filtergrenzen des verwendeten Oktavsiebs sind in Tab. 1 angegeben. Dabei ist zu berücksichtigen, daß besonders in den hohen Siebbereichen mehrere Teiltöne in eine Oktave fallen. Rechnet man z. B. mit einem Grundton von $65 \mathrm{~Hz}$, dann liegen in Sieb I, 1 der Grundton, in I, 2 der 2. und 3. Teilton, in I, 3 drei Teiltöne (4., 5., 6.), in I, 4 sechs Teiltöne (7. bis 12.), in I, 5 zwölf Teiltöne (13. bis 24.) usw. In den Sieben der Umschaltung II verläuft die Entwicklung etwas anders: In II, 1 liegt ein Teilton (1.), in II, 2 ein Teilton (2.), in II, 3 zwei Teiltöne $(3 ., 4$.), in II, 4 vier Teiltöne (5. bis 8.), in II, 5 neun Teiltöne (9. bis 17.) usw. An der Zahl der Teiltöne pro Sieb ändert sich nichts, wenn die Grundtöne der Klänge eine oder mehrere Oktaven höher liegen. Der Grundton erscheint dann in einem anderen Bereich und die Obertöne folgen in der angegebenen Verteilung in den höheren Sieben.

Als auffallendes Charakteristikum des Vollen Orgelklangs ergab sich, daß die Teiltöne der 3., 5., 7., 9., 11., 13. usw. Ordnung schwächer sind als die anderen. Wenn man dies berücksichtigt und dazu noch die Zahl der Obertöne pro Oktave kennt, so gewinnt man trotz der geringen Auflösung der Oktavsiebanalyse ein in den meisten Fällen ausreichendes Bild von der Intensitätsverteilung im Spektrum der Plenumklänge.

Das Mikrophon befand sich bei den Aufnahmen der Klänge an denselben Stellen, wo auch der Schalldruck gemessen wurde ${ }^{1}$. Eine Darstellung der Spektren im Schalldruckmaß schien deshalb nicht notwendig zu sein. Alle in vorliegender Arbeit gezeigten Spektren sind daher im \%-Maßstab angegeben. Bei den Magnetophonaufnahmen wurden von den Registern und ihren wichtigen Kombinationen 5 sec lang alle $C$ und Cis (zur Vervollständigung zuweilen auch Fis und $G$ ) angeschlagen. Dadurch wurden bei einer Bandgeschwindigkeit von $77 \mathrm{~cm} / \mathrm{sec}$ von jedem Klang $385 \mathrm{~cm}$ lange Streifen gewonnen, aus denen $1 \mathrm{sec}$ dauernde Stücke ausgeschnitten und zu Ringen geklebt wurden. Spielt man diese Ringe ab, so formt man den nur 1 sec währenden Vorgang zu einem dauernden um. Dadurch ist genügend Zeit gewonnen, die Analyse mit Genauigkeit durchzuführen.

Bei dem beschriebenen Vorgehen werden die raumakustischen Einflüsse auf die Größe der Teiltonintensität

4 M. Grützm a cher, ENT 4, 533 [1927].

5 G. B o s s e, Funk u. Ton 2 [1948]. Für leihweise Überlassung eines Oktavsiebs der Fa. Wandel u. Goltermann, Reutlingen, danke ich dieser Firma herzlich. 
mit erfaßt, d. h. die sich bei langem Tönen ausbildenden, stehenden Wellen verursachen eine Ortsabhängigkeit der Teiltonintensität. Um eine Anschauung darüber zu bekommen, in welchem Grade sich die Intensität mit dem Ort verändert, wurde bei einigen Versuchsreihen das Mikrophon bei tönender Pfeife in verschiedenen Richtungen etwa $10 \mathrm{~m}$ weit vom Aufnahmeort hin- und hergefahren. Dabei zeigte sich, daß der Schalldruck eines eine Kopfbewegung, um dasselbe zu erreichen. Hinter Säulen und Pfeilern ist die Schallintensität meist kleiner, bescnders bei den höheren Frequenzen. Beim Anhören von Orgelmusik sollte man also freie Sicht auf das Pfeifenwerk haben.

Wichtig ist der Befund, daß die harmonisch liegenden Teiltöne aufeinanderfolgender Ordnungen durch raumakustische Einflüsse nicht gleichsinnig beeinflußt werden,

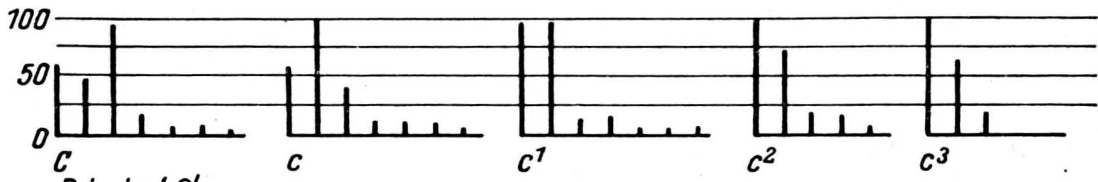

Prinzipal $8^{\prime}$

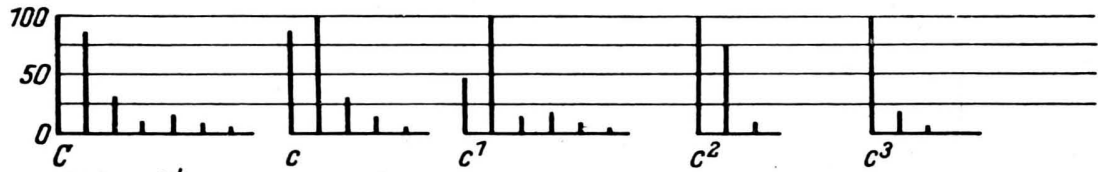

Oktave $4^{\prime}$

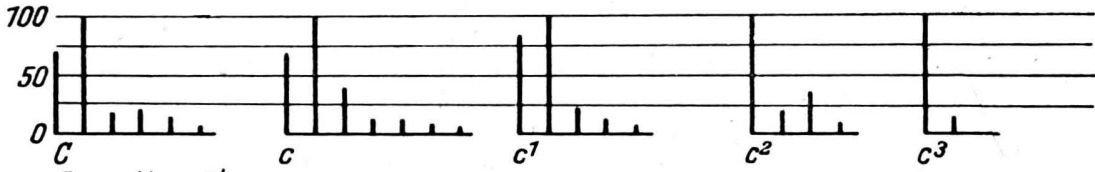

Superoktave $2^{\prime}$

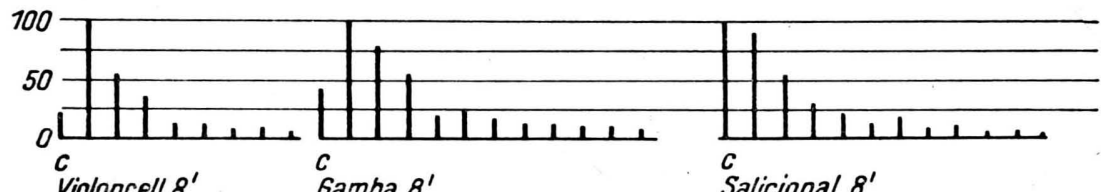

Violoncell $8^{\prime} \quad$ Gamba $8^{\prime} \quad$ Salicional $8^{\prime}$
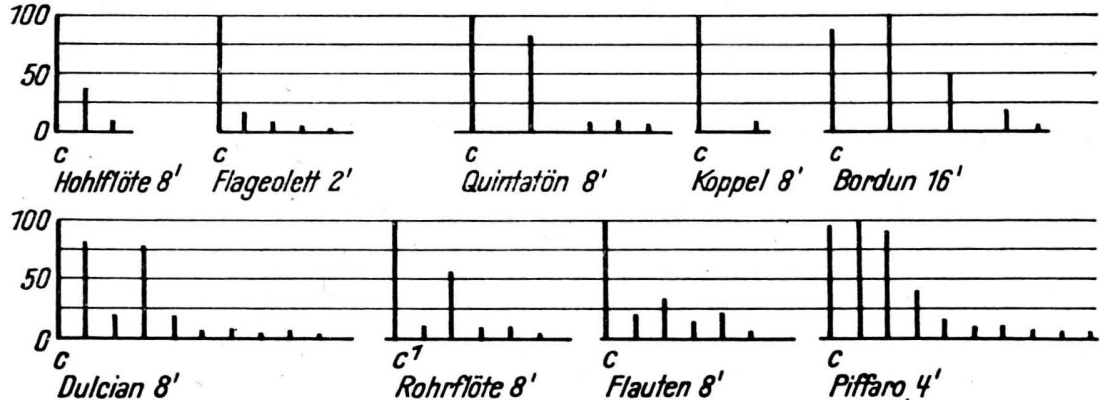

Abb. 1. Klangspektren von Labialpfeifen verșchiedener Register der Gabler-Orgel von Ochsenhausen. Messung im Raume.

Teiltons maximal bis auf $20 \%$ des größten gemessenen Wertes zurückgehen kann. Eine Auslöschung von 100\% war in keinem Falle nachzuweisen. Die Wahrscheinlichkeit, daß sich die von der Schallquelle und von allen Seiten einfallenden, reflektierten Wellen gerade zu Null ergänzen, ist sehr gering. Die Abstände der Orte größter Intensität sind abhängig von der Wellenlänge $\lambda$, wenn auch $\lambda / 2$ nicht die häufigste Größe dieser Entfernung ist. Während man bei tiefen Frequenzen seinen Standort um einige Meter verändern muß, wenn man eine Intensitätsänderung wahrnehmen will, genügt bei hohen Frequenzen

6 s. a. E. Thienha u s u. W. Will 1 s, Musik u. Kirche 5, 199 [1933]. so' daß auch der subjektive Charakter der Klänge im wesentlichen erhalten bleibt. Es kommt zwar vor, daß ein oder der andere Teilton eines Formantgebiets geschwächt, ein anderer verstärkt wird; das ganze Gebiet aber fällt nicht aus 7 .

\section{Me $\beta$ ergebnis se}

In Abb. 1 und 3 wird eine Auswahl typischer Klangspektren von Einzelpfeifen verschiedener Regi-

7 Über die relativ geringe Empfindlichkeit des Ohres für Teiltonunterschiede s. S. N a h r g a n g, Akust. Z. 3, 284 [1938]; E. Lö b, Akust. Z. 6, 279 [1941]. 
ster gezeigt, die an der Ochsenhausener Gabler-Orgel gewonnen wurden. Der Meßplatz befand sich etwa $30 \mathrm{~m}$ von der Orgel entfernt im Schiff der Kirche. Die Intensität des stärksten Teiltons ist überall gleich 100 gesetzt und die harmonisch liegenden Teiltöne sind in linearem $\mathrm{Ma}$ nach rechts in gleichem $\mathrm{Ab}$ stand aufgetragen. Die oberen drei Zeilen (Abb. 1) zeigen die Hauptregister der Orgel, die mit offenen Pfeifen versehen sind. Ihr Durchmesser nimmt, bezogen auf gleiche Grundtonhöhe, vom $8^{\prime}$ zum $2^{\prime}$ ab ${ }^{1}$. Man erkennt deutlich, daß derartige Pfeifen eine vollständige Teiltonreihe abgeben, daß sie in den Baßlagen obertonreicher sind als in den hohen Lagen und daß der erste und zweite Teilton die stärksten im Klange sind. Hervorgerufen werden diese Eigenschaften durch die Bauart (Mensur: Durchmesser, Aufschnitt usw.) und den anblasenden Wind. Es könnte der Gedanke auftauchen, warum die Pfeifen eines Registers nicht in verschiedenen Höhen das gleiche Klangspektrum besitzen. Wollte man dies erreichen, so müßten die Pfeifen der tiefen Lagen eine wesentlich größere Weite bzw. die der oberen Lagen eine geringere Weite erhalten. Ein musikalisches Bedürfnis für eine derartige Angleichung liegt offenbar nicht vor, im Gegenteil hat sich das Ohr daran gewöhnt bzw. ist so geartet, Spektren der gezeigten Zusammensetzung als im Charakter ähnlich zu empfinden. Interessant ist, daß die Klänge aller anderen Musikinstrumente das gleiche Verhalten zeigen ${ }^{\text {. }}$ Dasselbe wurde auch bei den Pfeifen anderer Register festgestellt, deshalb wurde bei diesen meist nur ein Beispiel (Spektrum der $c$-Pfeife) wiedergegeben. Man braucht sich nur vorstellen, daß die Klänge der höheren Lagen obertonärmer, die der tiefen obertonreicher als der dargestellte sind. Verringert man bei gleicher Grundfrequenz die Weite der Pfeifen, so wird der Klang unter sonst gleichen Bedingungen obertonreicher und bekommt dadurch leicht eine gewisse Schärfe und „streichenden“ Charakter. Beispiele solcher Stimmen liefert die vierte Zeile von Abb. 1. Im Bau derartiger "Streicher" hat sich Gabler eine besondere Meisterschaft angeeignet. Wenn auch das Charakteristikum wirklicher Streichinstrumentenklänge, nämlich das Hervortreten einiger Teiltöne, verursacht durch äußerst scharfe Resonanzen, der Natur nach nicht oder nur unvollkommen vorhanden ist, ist ihre Nachahmung, besonders durch die sehr zögernde Ansprache, gelungen. Wird die Weite der. Pfeifen größer als bei Prinzipal, so werden die Ober-

8 E. Meyer u. G. Buchmann, Abh. preuß. Akad. Viss., physik.-math. Kl. 1931, XXXII. töne schwächer, aber die Intensität des Grundtons wächst. Solche Spektren sind auf der zweiten Zeile von unten mit Hohlflöte und Flageolett veranschaulicht. Derartige Klänge wirken voll und weich und sind besonders zur Synthese von Klangfarben geeignet. In derselben Reihe sind die Spektren dreier gedackter Pfeifen gezeigt: Quintatön ist ein Register mit sehr geringer Weite, deshalb tritt der dritte Teilton, die Quinte über der Oktave, stark hervor. Überhaupt entfallen bei den Gedackten, wie bekannt, die geradzahligen Obertöne ganz oder treten nur als höhere Teiltöne in geringem Maß auf. Ist die Weite der Gedacktpfeifen sehr groß, so sind die Obertöne schwach und der Grundton intensiv, wie bei Koppel 8'. Bei Bordun sieht man das Spektrum einer Pfeife mit Grundton $65 \mathrm{~Hz}$. Auch hier treten die ungeradzahligen Obertöne hervor. Neben den genannten Stimmen gibt es noch solche mit konischen Körpern, und zwar solche, die sich nach dem Ende zu erweitern, wie Dulcian (untere Reihe links), bei dem deshalb die geradzahligen Obertöne überwiegen, und solche, die sich am Ende verjüngen. Das Spektrum von diesen unterscheidet sich wenig von den Pfeifen, die halbgedackt sind, die also am oberen Ende eine durchbohrte Kappe besitzen. Die entstehende Öffnung ist meist mit einem Röhrchen versehen. Bei derartigen Pfeifen sind die ungeradzahligen Teiltöne stärker als die geradzahligen, wie Rohrflöte und Flauten beweisen. Piffaro 4' (unten rechts) besteht aus zwei Pfeifen (Viola und Gedacktregister). Daher sind die ersten Teiltöne besonders stark.

Für die Möglichkeit, mit Hilfe solcher in Grundton- $\left(8^{\prime}\right)$ und anderen Lagen $\left(4^{\prime}, 2^{\prime}, 2^{2} / 3^{\prime}\right.$ usw.) befindlichen Spektren verschiedene Klangmischungen herzustellen, ist folgendes wesentlich: In den Jahren um 1910 wurden die Labialpfeifen mit so hohem Druck angeblasen, daß sie bei entsprechender Intonation wesentlich obertonreichere Klänge lieferten als die hier gezeigten ${ }^{9}$. Die Nachteile derartiger Klänge beruhen darauf, daß sie durchdringend und scharf, aber nicht farbig wegen des Fehlens der Formanten, wirken. Außerdem schließt die starke Obertönigkeit die Möglichkeit aus, von einander unterschiedene Klänge zu erzeugen. Dies soll Abb. 2 erläutern. Die effektive Zusammensetzung des Prinzipal und der Quinte ist bei hohem Druck links (a) ohne große Wirkung, während bei (b) im Betrieb mit schwachem Druck ein ganz neues Klangbild zustande kommt.

9 s. dazu die Spektren, W. Lottermoser, Akust. Z. 5, 329 [1940]; C. P. B ou er, J. Acous. Soc. Amer. 10, 32 [1938]. 
Die beste Bestätigung für die Richtigkeit dieser Anschauungen liefern die Riepp-Orgeln in Ottobeuren, bei der noch obertonärmere Labialstimmen als bei der Gabler-Orgel festgestellt wurden. Da Riepp aber auf jedem Manual eine Reihe von Aliquotstimmen (also künstliche Obertonstimmen) gebaut hat, bieten diese Orgeln geradezu erstaunliche Möglichkeiten der Klangsynthese ${ }^{10}$. Es dürfte danach in jeder Hinsicht als günstig zu erachten sein, die moderne Orgel unter ähnlichen Bedingungen wie die Barockorgeln zu betreiben.

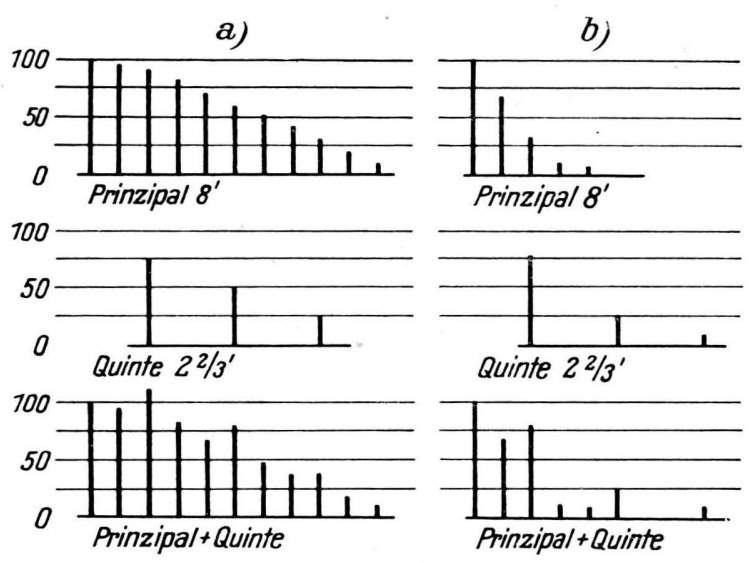

Abb. 2. Zusammensetzung einer Grund-mit einer Aliquotstimme: a) bei starkem Winddruck, b) bei schwachem Winddruck.

Einige Spektren von Zungenpfeifen, die bekanntlich sehr viel obertonreicher sind, zeigt Abb. 3. Sie sind deswegen so interessant, weil bei ihnen deutliche Formanten auftreten, die durch die Hohlräume der Koustruktion bedingt sind und deshalb den Klängen einen vokalähnlichen Charakter verleihen. So sieht man bei Vox humana $C$ das Formantgebiet des "o", daneben ist der Näselformant ausgebildet. Deswegen wirkt der Klang stark nasal. Bei der $c$-Pfeife ist dasselbe der Fall. Bei $c^{1}$ erklingt ein dem „e“ ähnlicher Laut. Auch die $C$ - und $c$-Pfeifen der Schalmei liefern einen näselnden Klang, wie es auch im Wesen des gleichnamigen Blasinstruments begründet ist. Das Spektrum der Hautbois-Stimme deutet auf einen hellen „e"-Klang. Die ganze Stimme ist unserer Orchesteroboe außerordentlich ähnlich.

Die Trompete unterscheidet sich von den ebengenannten Zungenpfeifen vor allem durch ihre größere Intensität. Ihre Pfeifen sind mit großen, koni-

10 Ich bin dem Organisten, Hrn. Dr. Greß, Ottobeuren, zu Dank verpflichtet, der mir die schönsten Klangfarben der Orgel vorführte. schen Schallbechern versehen, welche die Teiltöne des o- und a-Formanten besonders hervortreten lassen. Die Pfeifen sind frei von näselndem Charakter, indessen wurden bei anderen Orgeln Trompeten gemessen, welche in ihrem Spektrum zum Nachteil ihrer Wirkung den Näselformanten besaßen. Die Spektren der Posaune des Pedals waren ähnlich wie die gezeigten Trompetenspektren.

Wie durch die Analysen besonders deutlich erwiesen wurde, sind die gemischten Stimmen akustisch von ganz besonders großer Bedeutung. Man rechnet unter diese, um nur die Hauptvertreter zu nennen: Mixturen, Zimbeln und Kornette. Letztere sollen hier außer acht bleiben, weil sie infolge ihrer Zusammensetzung typische Solostimmen sind und zum Gesamtklang nicht beigezogen werden. Gemischte Stimmen sind so angelegt, daß beim Druck einer Taste ein ganzer Pfeifenchor ertönt, also je nach Disposition meist 3 bis 8 Pfeifen zugleich. Ihre Grundfrequenzen liegen dort, wo sich auch die harmonischen Oktav- und Quintteiltöne der Pfeifen in Normallage $8^{\prime}$ oder auch $16^{\prime}$ befinden. Es werden also der 2., 3., 4., 6., 8., 12. usw. Teilton durch diese Pfeifen erzeugt bzw. verstärkt. In der Orgelbausprache heißt das, es werden Pfeifen mit folgenden Fußzahlen ${ }^{1}$ disponiert: zum $8^{\prime}: 4^{\prime}, 2^{2} /{ }^{\prime}, 2^{\prime}, 1^{1}{ }^{\prime}{ }^{\prime}, 1^{\prime}, 1 / 3^{\prime}$; zum $16^{\prime}$ : $5 \frac{1}{3}, 4^{\prime}, 2 \%{ }^{\prime}$ usw. Die Pfeifen werden ihrer Bestimmung gemäß rein zur Normallage gestimmt und verschmelzen bei guter Bauart vollständig mit dieser. Im Falle der Mixturen erzeugt man auf die beschriebene Art die Teiltöne der Mittel- und hohen Lagen, im Falle der Zimbeln die der hohen Lagen. Bei den Kornetten fügt man zu Quinten und Oktaven nod die Terz. Weil man die Chöre der gemischten Stimmen mit offehen Pfeifen besetzt, liefern diese naturgemäß selbst wieder Obertöne. Man kann sich aber leicht ausrechnen, daß diese ebenfalls harmonisch zum Grundton der Normallage liegen. Die Zusammensetzungen der Mixturen wurde bereits in dem ersten Teil der Arbeit ${ }^{1}$ auf Seite 302, 304 und 306 angegeben. Rechnet man die Fußzahlen in $\mathrm{Hz}$ um, so wird ersichtlich, daß die Grundtöne der Chöre nicht in demselben Verhältnis wie die Grundtöne der Normallage längs der Tonskala auswärtsrücken, sondern in tiefere Lagen zurückspringen. Man nennt diese Art der Frequenzverschiebung: Repetition. Es leuchtet ein, daß dadurch gewisse Frequenzbereiche betont werden und im Spektrum ähnlich in Erscheinung treten können wie ein Formant.

Wie die Abb. $4 \mathrm{a}$ und $\mathrm{b}$ veranschaulichen, dienen die gemischten Stimmen bei entsprechender Intona- 
tion dazu, Formanten, in weitem Sinne des Wortes, $\mathrm{zu}$ erzeugen ${ }^{11}$. Meist, wie hier, handelt es sich um wandernde Formanten, denn der betonte Frequenzbereich rückt mit wachsender Grundtonhöhe nach höheren Frequenzen. Es kommen aber auch, insbe-
Siebmitten $f_{\mathrm{m}}=/ f_{\mathrm{u}} f_{\mathrm{o}}$ die hinter den Oktavsieben auftretenden Spannungen aufgetragen wurden. Die größte wurde wieder gleich 100 gesetzt.

Es ist erstaunlich, daß die Orgelbauer des Barock und die früherer Zeiten die gemischten Stimmen be-
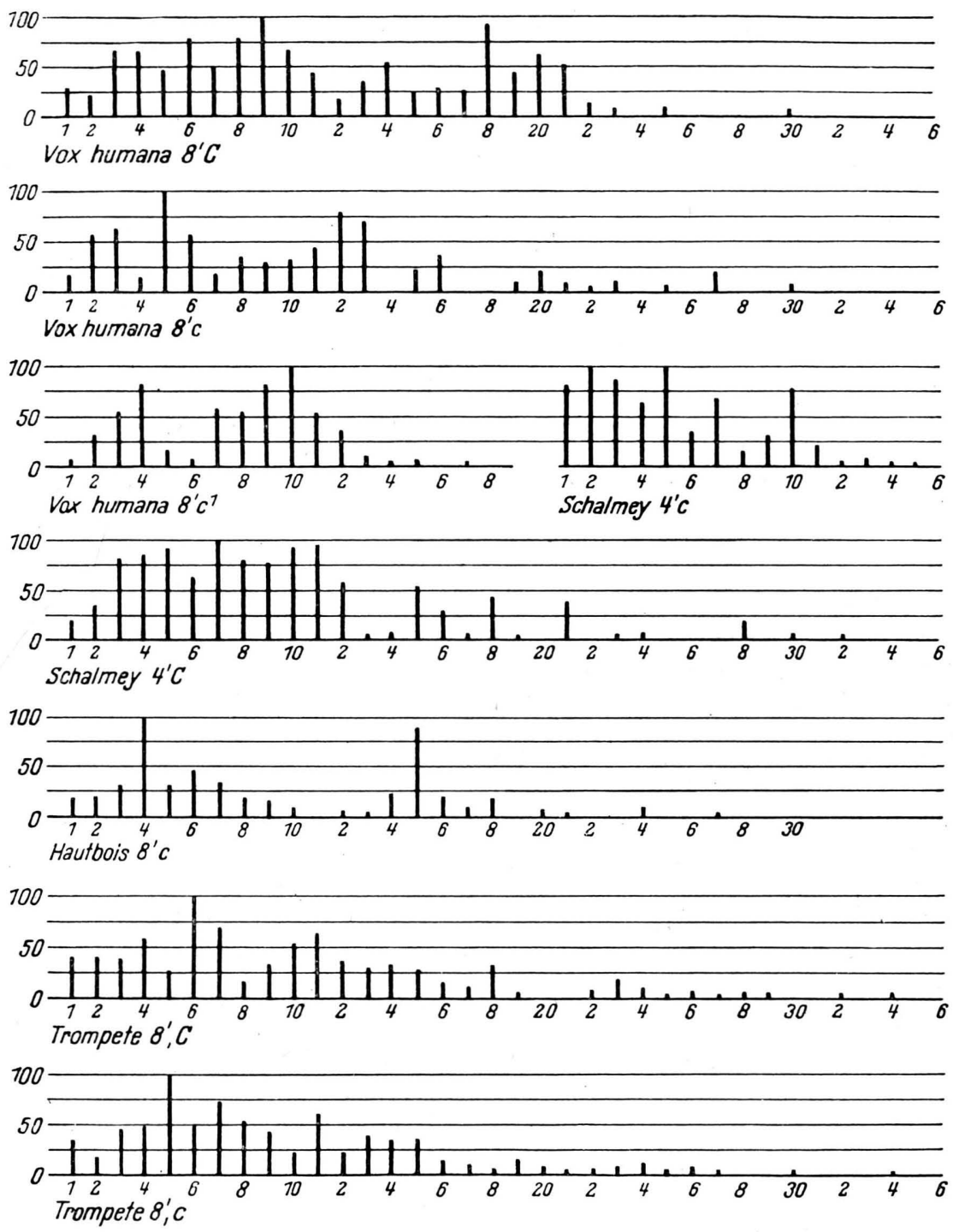

Abb. 3. Klangspektren von Zungenpfeifen verschiedener Register der Gabler-Orgel von Ochsenhausen. Messung im Raume.

sondere bei Zimbeln, feste Formanten vor, bei welchen der Frequenzbereich bei wachsender Grundtonhöhe konstant bleibt. Die Kurven der Abb. 4 und 5 wurden so gewonnen, daß bei den Frequenzen der

11 Auf die Zusammenhänge Mixtur-Formant weist schon Ch. Mahrenholz hin in seinem Werk: Die Orgelregister, Bärenreiter-Verlag, Kassel. reits in der dargestellten Form bauten, obwohl damals der Begriff des Formanten überhaupt nicht bekannt war. Sie besetzten außerdem Frequenzen, die sie besonders hervorheben wollten, durch zwei oder mehr Pfeifen mit gleicher Grundtonhöhe und erhielten dadurch Schwebungen, für die das Ohr besonders empfänglich ist, wie von der Wirkung der Zwei- 
und Dreifachsaiten beim Klavier bekannt ist. Der flachere Abfall der Kurven nach hohen Frequenzen rührt von den Obertönen der Pfeifen her. Die Mixtur Abb. 4 a erzeugt bei $C$ Teiltöne im Gebiete des uund o-Formants, liegt also relativ tief. Bereits bei $c$ liegt aber die Hauptintensität im o- und a-Bereich, um bei $c^{1}$ und $c^{2}$ ganz in den a-Bereich überzugehen. Während bei $c^{2}$ schon der e-Formant mit erfaßt wird, greift der Chor bei $c^{3}$ vom a- bis in den $i$-Bereich. in Frequenzgebiete bei $4000 \mathrm{~Hz}$. Die Mixturen der Holzhay-Orgel zeigen ein ganz anderes Verhalten. Hier setzt mit $C$ die Betonung des o-Formanten ein, die dunkle Färbung der Riepp-Hauptwerksmixtur fehlt hier. Bei $c$ wird der a-Formant hervorgebracht, bei $c^{1}$ der a- und e-Formant und schließlich erreicht mit $c^{3}$ die Helligkeit ihren höchsten Grad. Merkwürdigerweise liegen die Teiltöne der Zimbel Holzhays anfangs sehr tief und wandern über die Ton-
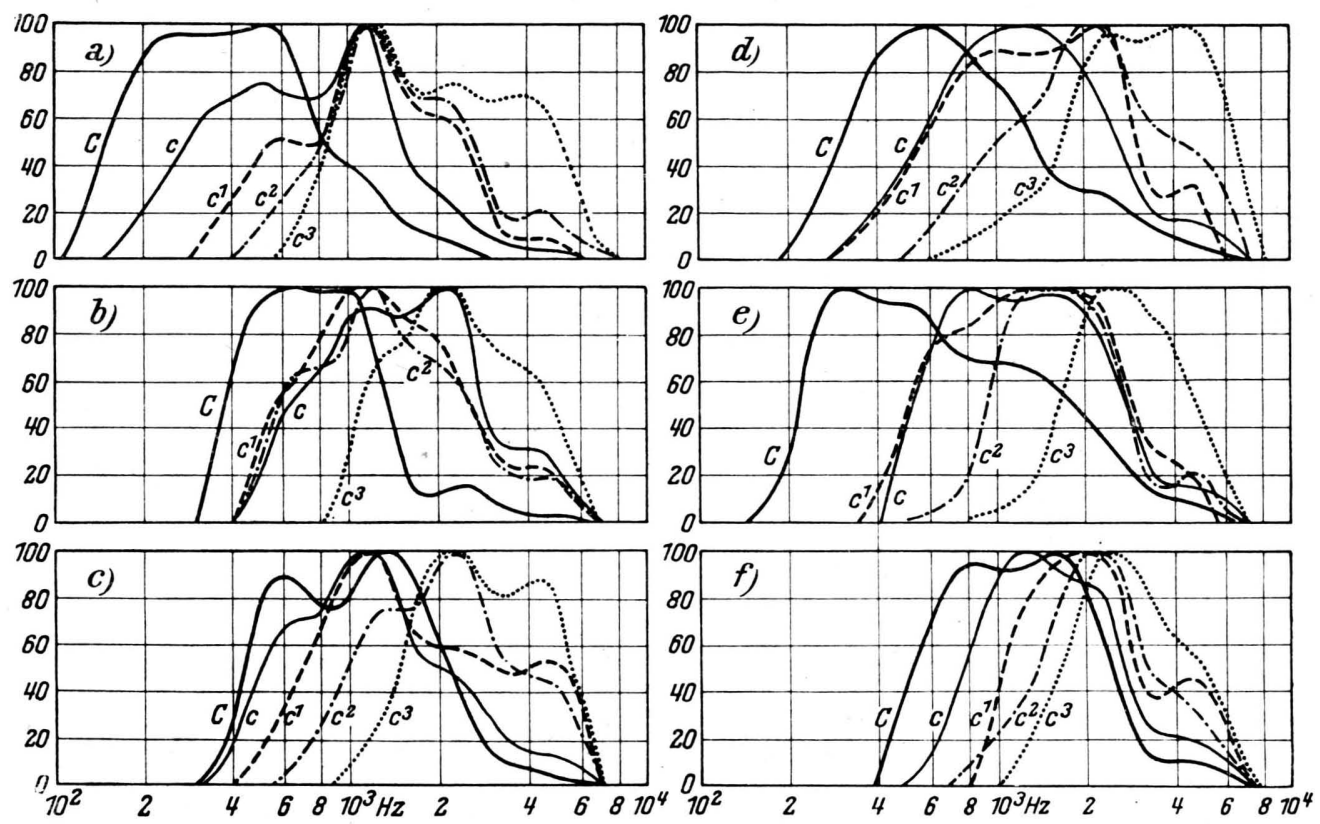

Abb. 4. Oktavsiebanalysen der gemischten Stimmen bei den Orgeln von Riepp (a, b, c, f) und Holzhay (d, e). Riepp-Gr. Orgel, Ottobeuren: a) HW, Mixtur 4-fach; b) HW, Zimbel 4-6-fach; c) Positiv, Formit 5-6-fach. Holzhay - Orgel, Obermarchtal: d) HW, Mixtur 5-fach; e) Positiv, Zimbel 5-fach; f) Riepp-Kl. Orgel, Ottobeuren: HW, Zimbel, 3-fach. An der Abszisse: Lage der Teiltöne.

Etwas geringer ist die Spanne bei der Zimbel. Hier liegen bei $C$ die Teiltöne im o- und a-Formant und wechseln in den höheren Lagen sofort in den a- und e-Bereich über. Bei $c^{3}$ ist sogar nur der e- und i-Bereich besetzt. An sich wäre die Aufgabe einer Zimbel die Betonung des e- und i-Formanten, wenn nicht nur des i-Formanten, was durch eine starke Repetition bei geringer Formantwanderung erreicht würde. Tatsächlich gibt es derartige Zimbeln, die den Klängen ein helles, schellenartiges Klingen beigeben. Die Zimbel Riepps und auch die Holzhays sind eigentlich Ergänzungen zu Mixturen bzw. hochliegende Mixturen. Noch höher als die genannten Stimmen reicht frequenzmäßig die Fourniture (Fornit genannt) von Ottobeuren. Auch die Besetzung bei $C$ liegt schon höher als bei der Zimbel und wandert bis $c^{3}$ skala nur bis zur Betonung der e-Lage. Diese ZimbeI trägt also ihren Namen zu Unrecht, obwohl sie klanglich hervorragend wirkt.

Wie eine geringe Formantwanderung zustande kommt, soll die Zimbel der kleinen Riepp-Orgel veranschaulichen. Bei dieser ist erreicht, daß im wesentlichen nur der a- und e-Formant erzeugt werden. Leider können aus der großen Zahl der Meßergebnisse nur diese wenige wiedergegeben werden. Natürlich muß außer der richtigen Frequenz der Einzelpfeifen das richtige Stärkeverhältnis zu den Grundstimmen gewahrt sein, damit die Mixturen die ähnliche Wirkung haben wie die gezeigten.

Wie schon in der Einleitung zum ersten Teil erwähnt wurde, kommt die Klangschönheit der Barockorgeln vor allem im Vollen Werk bzw. im Plenum zur 
Geltung. Letzteres erklingt dann, wenn die Prinzipale, Oktaven und Mixturen, zur weiteren Steigerung auch noch Bordun und die Trompeten und Posaunen gezogen sind. Stimmen dieser Art weist jede größere Orgel auf. Ihre gemeinsame Klangwirkung bei Frequenzen von etwa 400 bis $1000 \mathrm{~Hz}$ liegt, und zwar unabhängig von der Grundtonhöhe. Dieses Maximum wird hervorgerufen durch eine entsprechende Verteilung der beteiligten Teiltöne von Grund- und Oktavstimmen sowie der Mixturen. Bei
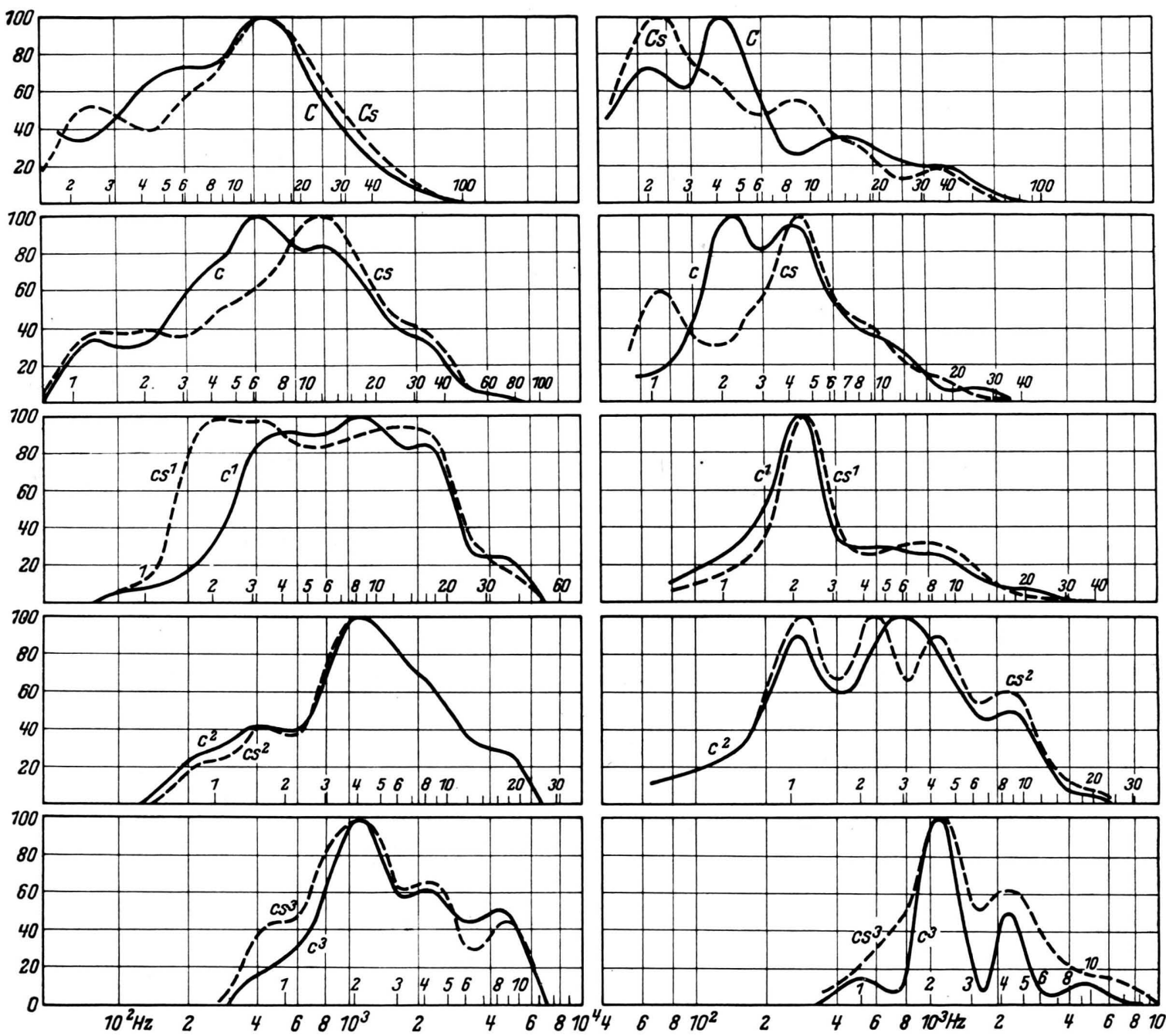

a)

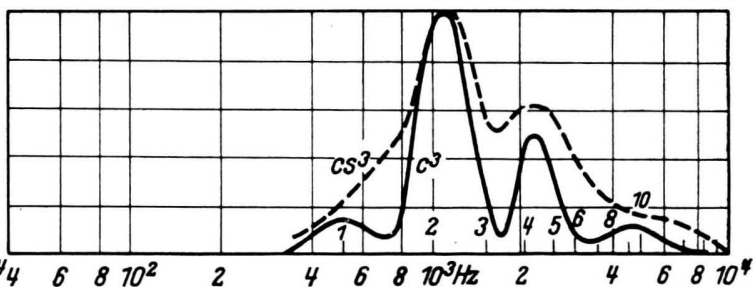

b)

Abb. 5. Oktavsiebanalysen der Plenumklänge des Hauptwerks a) der Großen Orgel von Riepp. Beteiligte Register: Copel 16', Prinzipal 8', Copel 8', Prinzipal 4', Mixtur, Zimbel. b) einer modernen Orgel. Beteiligte Register: Prinzipal 16', Prinzipal 8', Oktave 4', Oktave 2', Mixtur.

zur objektiven Gütebeurteilung einer Orgel heranzuziehen, erscheint durchaus sinnvoll. In den Abb. 5 a und $\mathrm{b}$ sind Oktavsiebanalysen von Plenumklängen der Riepp-Orgel und von denen eines modernen Instruments ungefähr gleicher Registerzahi dargestellt. Dabei erkennt man ohne weiteres, daß das Intensitätsmaximum bei den Klängen der Barockorgeln den Klängen mit tiefen Grundtönen sind es meist Teiltöne vom 8 . ab, die besonders stark sind, bei den höheren Grundtönen rücken der 1., 2. oder 4. an die bevorzugten Stellen. Dadurch werden die Formantbereiche des o und a, bei den höheren Klängen die des e und i betont. Durch Vergleich mit den vollständig anders verlaufenden Verteilungskurven der 
modernen Orgel (Abb. 5b) kann man ableiten, daß für die hervorragende Klangwirkung der Barockorgeln die wiedergegebene Intensitätsverteilung von wesentlicher Bedeutung ist. Nur so entstehen die ausgeglichenen, weder zu dunkel noch zu hell wirkenden Klänge, deren Farbe sich längs der Tonskala organisch vom o, über a und e zum i-Charakter entwickelt.

Um den Einfluß der Intonation der Pfeifen und der Raumakustik abschätzen zu können, wurden neben den $C$-Klängen auch die von $C$ is wieder- zwischen 2 und $4 \mathrm{kHz}$, die Aufhellung ist deshalb zu gering. Es ist offenbar für den Orgelbauer sehr schwer, bei verschiedenen raumakustischen Verhältnissen die richtige Mensur und Intonation zu finden, welche die günstige, spektrale Intensitätsverteilung bewirkt.

Zieht man zum Plenum die Zungenregister wie Trompeten und Posaunen, so ändert sich das Aussehen der Oktavsieb-Spektogramme bei den Barockorgeln nur ganz unwesentlich. Dies rührt von der relativ geringen Stärke und auffallend großen Obertönigkeit dieser Stimmen her.

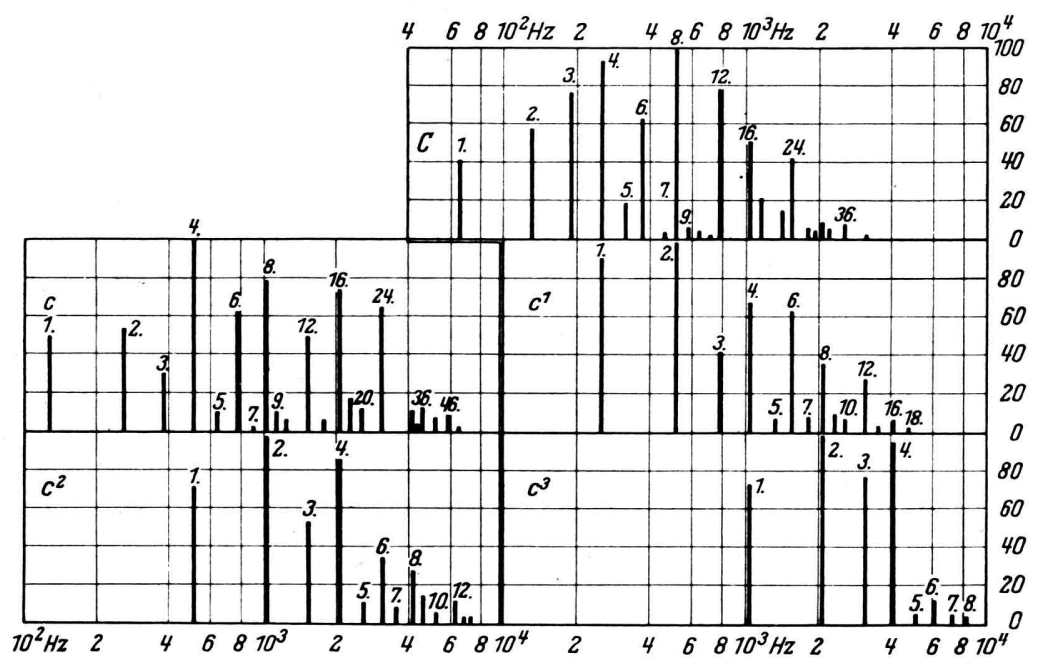

Abb. 6. Klangspektren des Plenums von dem Hauptwerk der Gabler-Orgel, Ochsenhausen. Prinzipal 8', Oktav 4', 2', Mixtur.

gegeben. Man erkennt daraus, daß oft beträchtliche Verschiebungen im Obertongehalt stattfinden, ohne daß jedoch das Intensitätsmaximum sich ausschlaggebend verändert. Es sei nur als Beispiel herausgegriffen, daß bei $c^{2}$ der Holzhay-Orgel das Maximum im o-Bereich, bei cis im a-Bereich liegt. Trotz dieser Verschiebung, die am Ort der Beobachtung tatsächlich einen Klangfarbenwechsel hervorruft, bleibt die als günstig erkannte Intensitätsverteilung im groBen und ganzen bestehen. Auf Abb. $5 \mathrm{~b}$ hat lediglich der $c^{2}$-Klang ein den Barockklängen ähnliches Aussehen. Allerdings müßte er nicht bei $c^{2}$, sondern bei $c^{1}$ erscheinen. Bei $C$ sieht man eine übermäßige Betonung tiefer Frequenzlagen, dasselbe gilt für $c$. Solche Klänge wirken dumpf und reizlos, vor allem dadurch ungünstig, daß sie außerdem noch übermäßig stark sind. Merkwürdig ist der $c^{1}$-Klang, bei dem eine Frequenz durch starke Teiltöne von Prinzipal $8^{\prime}$ und $16^{\prime}$ vorherrscht. Bei $c^{3}$ fehlen Teiltöne
Die als günstig erkannte Intensitätsverteilung wird im einzelnen durch die Spektren der Gabler-Orgel (Abb.6) bestätigt, wenn auch bei dieser das Maximum meist etwas tiefer liegt als bei den anderen Barockorgeln. Die Klänge der unteren Lagen wirken deshalb dunkler. Die Verteilung der Intensität auf die Teiltöne ist indessen derart, daß trotzdem hochwertige Klänge zustande kommen.

Besonders sei auf die für den Orgelklang, ganz allgemein, vorhandenen Unterschiede zwischen den einzelnen Teiltönen hingewiesen, die bei den Klängen von $c$ an deutlich herauskommen. Bei diesen ist der 3., 5., 7., 9. usw. Teilton immer schwächer als die übrigen, was eine Folge der am Klang beteiligten offenen Pfeifen im Oktav- und Quintabstand ist. Das Zurücktreten dieser Teiltöne bewirkt die klare, etwas strenge Klangfarbe, die jeder mit der Vorstellung von der Orgel verbindet.

Frühere Messungen an modernen Orgeln unter- 
schiedlicher Qualität ${ }^{12}$, bei denen eine große Zahl von Spektren aufgenommen wurden, hatten die gleichen Ergebnisse wie die vorliegenden. Als gute Bestätigung der Allgemeingültigkeit der als günstig erkannten Intensitätsverteilung ist weiterhin anzusehen, daß bei anderen wertvollen Instrumenten (wie z. B. den altitalienischen Meistergeigen ${ }^{13}$ ) die gleichen Erscheinungen zu beobachten sind. Auch da gilt scheinbar die bereits früher veröffentlichte Hüllkurve ${ }^{14}$ als

1. W. L o t t e rm o s e r, Instrumentenbau-Z. 3, 65 [1949].

13 H. B a ckhaus, Akust. Z. 4, 302 [1939]; H. Me in el, Akust. Z. 4, 89 [1939] u. vorhergehende Arbeiten.

14 W. Lottermoser, Physik. Bl. 3, 103 [1948]. eine Bedingung für eine befriedigende Klangwirkung.

Das Kultministerium des Landes Württemberg-Hohenzollern hat durch Bereitstellung von Mitteln über das Landesamt für Denkmalpflege die Durchführung dieser Arbeit ermöglicht. Dafür möchte ich auch hier meinen herzlichsten Dank aussprechen. Ebenso danke ich Hrm. Prof. Dr. Kossel für die freundliche Gewährung der Arbeitsmöglichkeit in seinem Institut und sein förderndes Interesse. Dank für jede Hilfe schulde ich auch den $\mathrm{Hw}$. Hrn. Pfarrern Scheel in Obermarchtal, Reich in Ochsenhausen, Pater Maurus in Ottobeuren sowie dem bekannten Orgelsachverständigen Hrn. Pfarrer Wör $\mathrm{s} \mathrm{ch}$ in g in Sandizell.

\title{
Über Addition von Maleinsäureanhydrid an phenylsubstituierte Anthracene
}

\author{
Von Hermann Hartmann und Heinz Grunert \\ Aus dem Institut für physikalische Chemie der Universität Frankfurt (Main) \\ (Z. Naturforschg. 5 a, 168-170 [1950]; eingegangen am 8. Juni 1949)
}

\begin{abstract}
Mit Hilfe der quantenmechanischen Theorie der aromatischen Verbindungen wird gezeigt, daß aus den neuen experimentellen Befunden von Gillet über die Addition von Maleinsäureanhydrid an 9.10-Diphenyl-anthracen und 9-Phenyl-anthracen auf eine Verdrehung der Phenylringe gegen das Anthracenskelett geschlossen werden kann.
\end{abstract}

$\mathrm{W}$ ir haben kürzlich die Anlagerungsreaktionen von Maleinsäureanhydrid an aromatische Kohlenwasserstoffe mit einer Anthracenkonfiguration vom Standpunkt der quantenmechanischen Theorie der aromatischen Verbindungen aus diskutiert ${ }^{1}$ und bei dieser Gelegenheit darauf hingewiesen, daß bei der Reaktion zwischen 9.10-Diphenyl-anthracen und Maleinsäureanhydrid eine grobe Diskrepanz zwischen der Theorie und den in der Literatur mitgeteilten Beobachtungstatsachen besteht. Wir hatten den (negativen) Anteil zur Wärmetönung der Anlagerungsreaktion berechnet, der von der Änderung des Sonderanteils der $\pi$-Elektronenenergie (d. h. von der Änderung der Resonanzenergie des $\pi$-Elektronensystems) bei der Anlagerung herrührt. Unter der Annahme, daß 9.10-Diphenyl-anthracen eben gebaut ist, erhielten wir für Addition in meso-Stellung $2,17 \beta$ ( $\beta$ bedeutet das Hückelsche Resonanzintegral). Demnach sollten für diesen Stoff die thermodynamischen Bedingungen für eine Addition noch ungünstiger sein als bei Benzol, während doch experimentell schon bei Naphthalin keine Addition mehr beobachtet wird. Da nun aber 9.10-Diphenyl-anthracen unter Bedingun-

: H. H a r t n a $n \mathrm{n}$. Z. Naturforschg. 3a, 29 [1948].

2 I. Gill e t, C. R. hebd. Séances Acad. Sci. 227, 853 [1948]. gen, unter denen z. B. Anthracen 99\% Anlagerungsprodukt liefert, noch zu $16 \%$ Maleinsäureanhydrid addiert, hatten wir vermutet, daß die Annahme einer ebenen Struktur des Kohlenwasserstoffes irrig ist. Tatsächlich kann man sich an einem Modell leicht klarmachen, daß die Wechselwirkung der am Kohlenstoffgerüst stehenden Wasserstoffatome eine streng ebene Struktur ausschließt, die beiden Phenylringe also aus der durch das Anthracenskelett bestimmten Ebene herausgedreht sein müssen. Eine solche Verdrehung würde nach der Theorie eine Verringerung der Resonanzenergie des 9.10-Diphenyl-anthracens und damit eine größere ,Aufspaltbarkeit“ des 7 Elektronensystems dieses Moleküls zur Folge haben.

Inzwischen hat aber nun I. G i 11 e $t^{2}$ nachgewiesen, daß 9.10-Diphenyl-anthracen Maleinsäureanhydrid nicht, wie bisher angenommen, in meso-Stellung, sondern in einem Seitenring addiert. Dies zeigt Abb. 1.

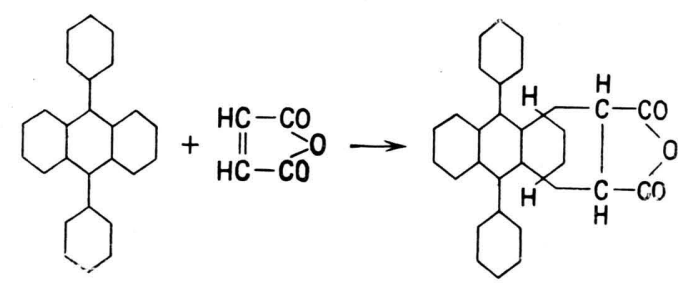

